Lily Gramatikov

Editorial

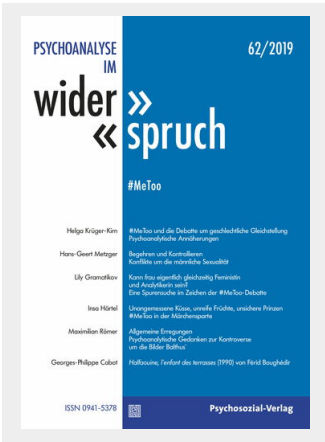

Psychoanalyse im Widerspruch

31. Jahrgang, Nr. 2, 2019, Seite 5-8

Psychosozial-Verlag

DOI: $10.30820 / 0941-5378-2019-2-5$

\title{
匫 Psychosozial-Verlag
}




\section{Impressum}

\section{Psychoanalyse im Widerspruch}

ISSN 0941-5378

31. Jahrgang, Nr. 62, 2019, Heft 2

https://doi.org/10.30820/0941-5378-2019-2

Herausgeber:

Institut für Psychoanalyse und Psychotherapie Heidelberg-Mannheim (IPP) und Heidelberger Institut für Tiefenpsychologie (HIT)

Redaktion:

Hans Becker, Helmut Däuker, Lily Gramatikov, Anja Guck-Nigrelli, Parfen Laszig, Sabine Metzger, Matthias Richter, Gerhard Schneider, Rolf Vogt

Leitender Redakteur:

Parfen Laszig

redaktion@parfen-laszig.de

Redaktionsadresse:

Institut für Psychoanalyse und Psychotherapie Heidelberg-Mannheim

Alte Bergheimerstraße 5

D-69115 Heidelberg

Telefon und Telefax: 062 21/18 4345

Manuskripte:

Die Redaktion lädt zur Einsendung von Manuskripten ein. Mit der Annahme des Manuskriptes erwirbt der Verlag das ausschließliche Verlagsrecht auch für etwaige spätere Veröffentlichungen.

Verlag:

Psychosozial-Verlag

Walltorstraße 10

D-35390 Gießen

Telefon: $0641 / 96997826$

Telefax: 06 41/96997819

bestellung@psychosozial-verlag.de
Bezug:

Jahresabo: 29,90 Euro (zzgl. Versand) Einzelheft: 19,90 Euro (zzgl. Versand) Studierende erhalten $25 \%$ Rabatt (gegen Nachweis).

Das Abonnement verlängert sich jeweils um ein Jahr, sofern nicht eine Abbestellung bis acht Wochen vor Beendigung des Bezugszeitraums erfolgt.

Bestellungen von Abonnements bitte an den Verlag, bestellung@psychosozial-verlag.de, Einzelbestellung beim Verlag oder über den Buchhandel.

Anzeigen:

anzeigen@psychosozial-verlag.de

Es gelten die Preise der aktuellen Mediadaten. Sie finden sie im Downloadbereich auf www.psychosozial-verlag.de.

Erscheinungsweise: Halbjährlich

Copyright:

(C) 2019 Psychosozial-Verlag, Gießen

Nachdruck - auch auszugsweise - mit Quellenangabe nur nach Rücksprache mit den Herausgebern und dem Verlag. Alle Rechte, auch die der Übersetzung, vorbehalten.

Satz:

metiTec-Software, me-ti GmbH, Berlin www.me-ti.de 


\section{Editorial}

Psychoanalyse im Widerspruch, Nr. 62, 31 (2) 2019, 5-8

https://doi.org/10.30820/0941-5378-2019-2-5

www.psychosozial-verlag.de/piwi

Diese Ausgabe der Zeitschrift Psychoanalyse im Widerspruch widmet sich einem gesellschaftlichen Thema mit erheblichen Konfliktpotential: der \#MeToo-Debatte. In den Jahren 2017 und 2018 liefen die medialen Kanäle heiß. Die unter dem Hashtag "MeToo " angezeigten Vorfälle von sexuellen Übergriffen zogen Untersuchungen und - in manchen Fällen - Suspendierungen oder gar Gerichtsprozesse nach sich. Diskussionen über die Glaubwürdigkeit der Berichte, über die Vorverurteilung durch die Berichterstattung, aber auch über die Sinnhaftigkeit der gesamten Debatte wurden erbittert ausgetragen. Die Beschuldigten, meist prominente Männer, führten Gegenkampagnen und beharrten auf ihrer tatsächlichen oder aber behaupteten Unschuld. Heute steht die Wort-Neuschöpfung MeToo als Stichwortgeberin immer dann bereit, wenn es um die sexuelle Ausbeutung von Abhängigkeitsbeziehungen oder um die mannigfaltigen Diskriminierungen im Alltag geht. \#MeToo berührt die Frage nach dem Verhältnis der Geschlechter zueinander und richtet dabei den Blick erneut auf den allgegenwärtigen Verhaltenscodex von Frauen und Männern, auf Unterwerfung und Macht, stellt das »Opfer Frau « einer »toxischen Männlichkeit « gegenüber. Es geht auch um die stillschweigenden Regeln, worüber gesprochen werden kann und was verschwiegen werden muss. Es wird gekämpft, und da, wo nicht gekämpft wird, geht es um die Anerkennung von Differenz und Fremdheit, um das Ringen um gegenseitige Akzeptanz und Respekt.

Was Männer und Frauen trennt und was sie verbindet, dazu sollte die Psychoanalyse doch einiges zu sagen haben. Und so versammelt dieser Band unterschiedliche Perspektiven auf das wiederkehrende Sujet, wie sich die Entwicklungslinien von »Männlichkeit « und »Weiblichkeit « psychoanalytisch denken lassen und auf welche Weise Gesellschaft, Kultur und Patriarchat auf das Unbewusste, auf die Beziehungsgestaltung und den künstlerischen Schaffensprozess Einfluss nehmen.

Das Heft beginnt mit einem grundlegenden Beitrag von Helga KrügerKirn, "\#MeToo und die Debatte um geschlechtliche Gleichstellung ", in welchem die Autorin der Frage nachgeht, wie es zu den »Beharrungstendenzen in den geschlechtlichen Kräfteverhältnissen " kommt. Dabei stellt sie die von ihr in der psychoanalytischen Behandlungssituation beobachtete Schwierigkeit einer weiblichen sexuellen Subjektivierung in Relation zu dem 
Paradoxon der begrenzenden und tabuisierenden gesellschaftlichen Blickweise auf den weiblichen Körper. Gerade die fortwährende sexualisierte Zurschaustellung stellt sich dabei als eine Einschränkung der Inbesitznahme des eigenen Körpers heraus. Die für eine gesellschaftlich akzeptierte Weiblichkeit geforderte Unterdrückung von libidinösem und aggressivem Begehren führe, so die Autorin, zu spezifischen Abwehrstrukturen, die sich ihrerseits negativ auf das Selbsterleben auswirkten. Für die Psychoanalyse fordert Krüger-Kirn eine kritische Reflexion der eigenen Theoreme und Paradigmen.

Während der Beitrag von Krüger-Kirn wesentlich die weibliche Entwicklung in den Blick nimmt, beschäftigt sich der darauffolgende Beitrag von Hans-Geert Metzger mit der » Krise der Männlichkeit «. Unter der Überschrift »Begehren und Kontrollieren - Konflikte um die männliche Sexualität « lotet der Autor das Spannungsverhältnis aus zwischen der feministischen Forderung nach einer Veränderung des klassischen Männlichkeitsbildes und der zu beobachtenden Wiedererstarkung der traditionellen männlichen Geschlechterrolle. Metzger untersucht, in wie weit die Angst des Mannes vor der Frau eine Folge der frühen kindlichen Abhängigkeitsbeziehung darstellt. In der erotischen Beziehung werde der Mann mit seiner internalisierten Mutterimago konfrontiert, die sich in ein begehrenswertes weibliches Objekt und ein übermächtiges mütterliches Objekt spalte. Der "phallische Monismus « sei auch eine Reaktion auf diese Angst und darüber hinaus ein Schutz gegen die Anerkennung der eigenen Unvollkommenheit. Werde diese Form der männlichen Abwehr überwertig, führe das in der Beziehung zu einer Frau zur Ausbildung von Machtstrukturen. Gleichzeitig stelle die Vorstellung einer ausschließlich aggressiven und triebgesteuerten männlichen Sexualität eine pervertierte Männlichkeit dar. Vereinigende Sexualität gehe hingegen mit einer Grenzüberschreitung einher, die allerdings gleichermaßen Lust und Angst auslöse.

In meinem eigenen Beitrag »Kann frau eigentlich gleichzeitig Feministin und Analytikerin sein? Eine Spurensuche im Zeichen der \#MeToo-Debatte « beziehe ich mich zunächst auf die lange Tradition der feministischen Kritik an der androzentristischen Prägung der psychoanalytischen Theorie. Dabei wird deutlich, dass im gegenwärtigen gendertheoretischen Diskurs die Frage der Ungleichheit der Geschlechter zu Gunsten eines Versprechens, das eigene Selbst individuell und jenseits der herrschenden Geschlechtergrenzen gestalten zu können, verloren geht. In der \#MeToo-Debatte wird jedoch meines Erachtens die bestehende patriarchale Ordnung erneut aufgedeckt. Zudem offenbart auch die medial geführte Diskussion über \#MeToo das patriarchal geprägte Geschlechterverhältnis. Aufgrund der feministischen Erkenntnis 
der Allgegenwärtigkeit der »heterosexuellen Matrix « ergibt sich für die psychoanalytische Behandlungssituation die Schwierigkeit, dass nicht nur die bewussten Kognitionen über die Behandlung oder die psychoanalytischen Theorien, sondern eben auch die unbewusste Vorstellungswelt untrennbar mit den patriarchalen Vorschreibungen verbunden sind. Ich plädiere deshalb für ein nicht-theoriegeleitetes »ungesättigtes analytisches Zuhören «, um jene Aspekte des Rapports, die sich außerhalb der herrschenden symbolischen Ordnung befinden, aufnehmen zu können.

Insa Härtel und Maximilian Römer befassen sich in ihren jeweiligen Arbeiten mit den kulturtheoretischen Implikationen der \#MeToo-Debatte bzw. den unter dem Argumentationsdach von \#MeToo geführten Angriffen bzgl. der potenziell diskriminierenden Aussagen kulturell-künstlerischer Werke. Härtels Beitrag »Unangemessene Küsse, unreife Früchte, unsichere Prinzen: \#MeToo in der Märchensparte " greift eine in England geführte Diskussion aus dem Jahr 2017 über ein Schulbuch für die ersten Klassen auf, in der es um die Darstellung des Märchens »Dornröschen « geht. Das Wachküssen der schlafenden Prinzessin durch den Prinzen sei - so die damalige Argumentationslinie - ein unangemessenes übergriffiges Verhalten, das jüngere Kinder nicht einzuordnen in der Lage wären. Härtel führt mit einer kenntnisreichen Analyse durch die Bedeutungsebenen der spezifischen Darstellung in dem angemahnten Schulbuch und zeigt auf diese Weise, wie die Argumentationsführenden durch den Mechanismus der Projektion den Wunsch nach einer eigenen "kindlich-unschuldigen Sphäre" auf das vermeintlich unschuldige Kind verschieben, um eine Grenze zu dem TriebhaftSexuellen zu etablieren. Der im kulturellen Erzeugnis des Schulbuchs bereits stattgefundene Aushandlungsprozess im Sinne einer Adaptierung an die aktuellen gesellschaftlichen Veränderungen werde zugunsten einer Täter-Opfer-Schablone negiert. Darin zeige sich die Vorstellung einer passiven kindlich-weiblichen Welt, in der die bedrohliche Sexualität im Außen verortet wird, das Subjekt von der Auseinandersetzung mit der Herausforderung des Sexuellen vermeintlich verschont bleiben kann.

In seinem Beitrag »Allgemeine Erregungen - Psychoanalytische Gedanken zur Kontroverse um die Bilder Balthus' " untersucht Maximilian Römer die in Zusammenhang mit der \#MeToo-Debatte erneut aufgeworfene Frage, ob das umstrittene künstlerische Werk des Malers Balthus nicht vorrangig den Voyeurismus der Zuschauenden im öffentlichen Raum des Museums befriedige. In der fraglichen Online-Petition an das MoMa, New York, wurde konkret das Bild Thérèse rêvant angemahnt, auf dem ein junges Mädchen in sitzender Haltung abgebildet ist. Skandalon dieses Gemäldes ist die zwischen dem weit fallenden Rock sichtbare Unterwäsche des Mäd- 
chens; ihr Schritt wird so den Betrachtenden freigegeben. Römer vollzieht die kunstgeschichtliche Debatte um Balthus' Werk nach und zeigt, wie sich im suchenden Verständnis des Werkes Pädophilie-Vorwürfe mit biografischen Erklärungsversuchen abwechseln. Im Rückgriff auf Freud und Laplanche stellt Römer die Betrachtung des Bildes ins Zentrum seiner Analyse. In Moment der Betrachtung fiele das äußere Bild mit eigenen inneren Bildern zusammen, sodass in Bezug auf das fragliche Werk ein Begehren aktualisiert werde, das wegen seiner Unvereinbarkeit mit dem übermächtigen Inzesttabu nicht anerkannt werden könne und deshalb projektiv im - dann als pädophil gekennzeichneten - Anderen untergebracht und dort bekämpft werden müsse.

Mit der sich anschließenden Analyse des Filmes Halfaouine, L'enfant des terrasses führt Georges-Philippe Cabot in die islamische Welt der Geschlechtertrennung und der damit einhergehenden Initiationsriten ein. Halfaouine, ein Viertel in Tunis, zeigt die Welt des jungen Protagonisten Noura, der - wie Cabot darstellt - an der Schwelle zwischen der mütterlichen Welt des Kindes und der männlichen Welt der Erwachsenen seine Sexualität entdeckt. In der arabischen Kultur mit ihren festgeschriebenen Rollenbildern entdeckt Cabot eine »identifikatorische Leere", die für die dortige männliche Entwicklung konstitutiv sei. Die unaufgelöste Ödipalität, die durch die Vorschriften des Patios und des Hammam im Realen in Szene gesetzt werde, führe zu einer Idealisierung der Frau. Gleichzeitig werde die Vertreibung des Sohnes aus der mütterlichen Sphäre aufgrund der Unerreichbarkeit der Väter nicht aufgefangen, so dass dieser ohne konkrete Identifikationsobjekte bleibe.

Das Heft endet mit zwei Buchbesprechungen und dem Kalender für die psychoanalytischen Filmveranstaltungen in Heidelberg und Mannheim.

Lily Gramatikov Die Redaktion 